\title{
Observatorio
}

\section{El factor de impacto de las revistas científicas: limitaciones e indicadores alternativos}

\author{
Por Rafael Aleixandre-Benavent, Juan Carlos Valderrama-Zurián y Gregorio González-Alcaide
}

Resumen: El factor de impacto mide la repercusión que ha tenido una revista en la literatura científica mediante el recuento de las citas que ha recibido. A pesar de sus limitaciones, las agencias de evaluación de algunos países, entre ellos España, lo utilizan en la valoración de la actividad investigadora, creando un profundo malestar en determinados círculos que consideran que su uso es inapropiado e indiscriminado. Como alternativa al factor de impacto de Thomson ISI se han propuesto otros indicadores alternativos, si bien ninguno de ellos ha alcanzado suficiente aceptación y difusión como para que se apliquen sistemáticamente. Entre las iniciativas españolas, debe destacarse el Factor de impacto potencial de las revistas médicas españolas, desarrollado por el grupo Siniac (Sistemas de información e indicadores de actividad científica) del Instituto de Historia de la Ciencia y Documentación López Piñero, de Valencia y el Índice de impacto de las revistas españolas de ciencias sociales (In-Recs), desarrollado por el grupo de investigación $E C^{3}$ (Evaluación de la ciencia y de la comunicación científica) del departamento de Biblioteconomía y Documentación de la Universidad de Granada.

Palabras clave: Indicadores bibliométricos, Índices de impacto, Evaluación científica

\section{Title: Scientific journals impact factor: limita- tions and alternative indicators}

Abstract: Impact Factor measures the average number of

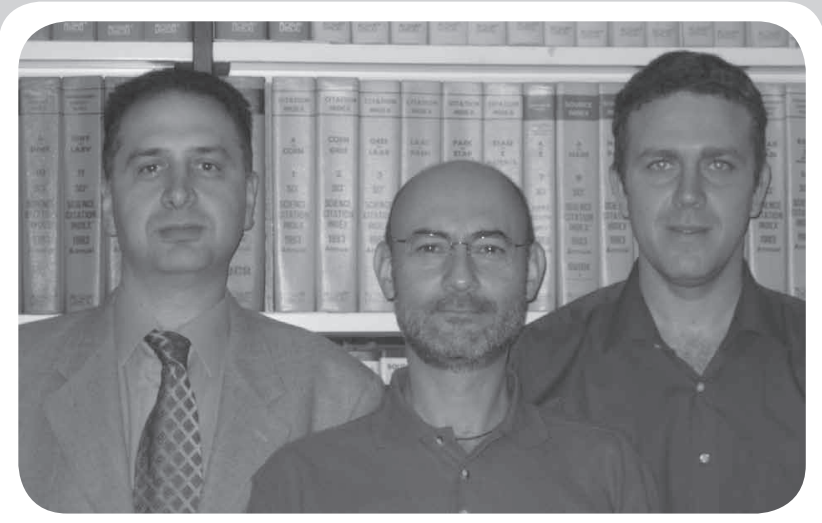

Juan Carlos Valderrama-Zurián es profesor titular de la Universitat de València, jefe de la unidad de Documentación del Instituto de Historia de la Ciencia y Documentación López Piñero y codirector de la base de datos IME y del proyecto Factor de impacto potencial de las revistas médicas españolas.

Rafael Aleixandre-Benavent es doctor en Medicina y científico titular del Consejo Superior de Investigaciones Científicas. Desde 2001 es codirector de la base de datos IME (Índice médico español) y del proyecto Factor de impacto potencial de las revistas médicas españolas.

Gregorio González Alcaide es licenciado en Historia y diplomado en Biblioteconomía y Documentación por la Universitat de València y Licenciado en Documentación por la Universidad Politécnica de Valencia. En la actualidad desempeña su actividad como investigador predoctoral del Consejo Superior de Investigaciones Científicas en el Instituto de Historia de la Ciencia y Documentación López Piñero.

times that articles published in the last two years are cited in the current year. In spite of its limitations, the evaluation agencies of some countries like Spain use it in research assessment, generating a deep unease within some circles that consider its use inappropriate and indiscriminate. As an alternative to the Impact Factor of Thomson ISI, other indicators have been proposed, although none of them have achieved sufficient acceptance and widespread use to be systematically applied. Among Spanish initiatives, most notable are the Potential Impact Factor of The Spanish Medical Journals, developed by the Siniac team (Information systems and research activity indicators) from the Instituto de Historia de la Ciencia y Documentación López Piñero (Valencia, Spain), and the Índice de impacto de las revistas españolas de ciencias sociales (In-Recs), developed by the EC $C^{3}$ research group at Library and Documentation department of Granada University (Spain).

Keywords: Bibliometric indicators, Impact factors, Research assessment

Aleixandre-Benavent, Rafael; Valderrama-Zurián, Juan Carlos; González-Alcaide, Gregorio. "El factor de impacto de las revistas científicas: limitaciones e indicadores alternativos". En: El profesional de la información, 2007, enerofebrero, v. 16, n. 1, pp. 4-11.

\section{Introducción}

El número de revistas científicas a nivel mundial se estima en más de 16.000 y no para de crecer. Sin embargo, no todas las revistas tienen la misma calidad, por lo que los profesionales de la información y docu- mentación científica, investigadores y responsables de la evaluación necesitan disponer de indicadores cuantitativos que midan su calidad y les faciliten la toma de decisiones en la selección de revistas para la formalización de suscripciones, el envío de manuscritos y la evaluación de la actividad científica ${ }^{1}$. 
El indicador bibliométrico más utilizado para estos fines es el factor de impacto (FI), ideado inicialmente por Eugene Garfield para identificar las revistas que debían formar parte de la cobertura del Science citation index $(S C I)$ y publicado anualmente en el Journal citation reports $(J C R)$. El FI de una revista es el número medio de veces que son citados en el año actual los artículos de esa revista publicados en los dos últimos años. Se calcula en cada revista estableciendo la relación entre las citas que en un año han recibido los trabajos publicados durante los dos años anteriores y el total de artículos publicados en ella durante esos dos años (por ejemplo, para calcular el factor de impacto de la revista Scientometrics en 2005 (FI=1,738), se suman todas las citas que han recibido en 2005 los trabajos publicados en 2004 y $2003(158+141)$, y se dividen por el número total de artículos publicados por dicha revista en 2004 y $2003(89+83)^{2,3}$.

La facilidad de consulta (desde todos los centros de investigación nacionales se puede acceder gratuitamente a la Web of Science gracias a la suscripción institucional realizada por la Fundación Española para la Ciencia y la Tecnología (Fecyt) y el Ministerio de Educación y Ciencia y la ausencia de otros instrumentos de medición ha favorecido que las agencias de evaluación de España (y de muchos otros países) lo utilicen en la valoración de las publicaciones y de la actividad investigadora de los científicos, grupos e instituciones. Por este motivo, muchos investigadores tratan de publicar sus trabajos en revistas que ocupen altas posiciones en las listas de categorías temáticas ordenadas por factor de impacto del JCR y lograr así un mayor reconocimiento de su labor ${ }^{3,4}$.

\section{http://www.accesowok.fecyt.es/}

Una medida relativa de la importancia del tema y del interés que despierta entre los investigadores es observar el número de artículos españoles publicados sobre el FI. Solamente en el área biomédica se han pu- blicado al menos 184 trabajos en los últimos 20 años, de los que el $80 \%$ son artículos originales en los que suelen identificarse, mediante estudios ad hoc, los flujos de citaciones que permiten conocer el FI de revistas específicas no incluidas en SCI. En otros trabajos se polemiza sobre los más variados aspectos relacionados con este tema: consejos para mejorar el FI, perspectivas, frustraciones, etc. ${ }^{5,6}$.

\section{"El factor de impacto del JCR tiene serios desequilibrios que cuestionan su validez cuando se utiliza en la evaluación de la actividad científica"}

\section{Limitaciones del análisis de citas y del FI}

Los análisis de citas, las bases de datos SCI y Social science citation index (SSCI) y el FI tienen unos inconvenientes que cuestionan seriamente su validez cuando se utilizan en la evaluación de la actividad científica, los cuales han sido ampliamente revisados en la literatura científica, tanto extranjera ${ }^{7-11}$ como nacional ${ }^{12-14}$.

A) Análisis de citas.

La moderna normativa científica exige que cuando un autor publica los resultados de un trabajo, se refiera a otros que lo han precedido. La función de las citas en la ciencia moderna es la de reconocer que los métodos, interpretaciones, datos, etc., proceden de los autores y publicaciones citadas ${ }^{15,16}$. Sin embargo, los trabajos son citados no sólo porque han influido sobre el trabajo que los cita, sino también por otras razones extracientíficas, entre las que pueden mencionarse las citas realizadas por formulismo sin ningún rigor, o para "salir del paso" y la existencia de citas negativas para

\section{Versión online de EPI}

Existe una versión electrónica de El profesional de la información, de uso gratuito para todos los suscriptores de la revista, que permite acceder a través de internet a los textos completos y materiales gráficos publicados desde el año 2000.

Más información en: http://www.elprofesionaldelainformacion.com/contenidos.html 


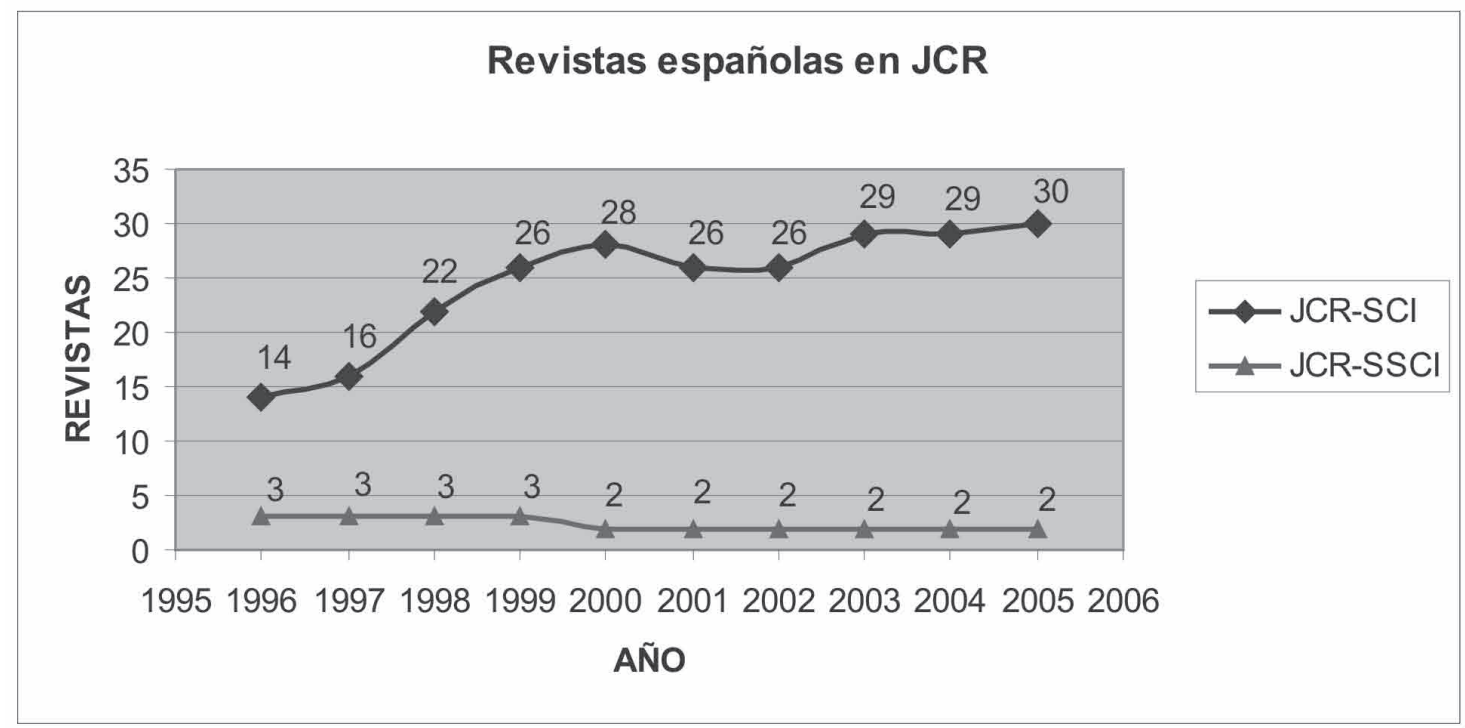

Figura 1. Evolución de las revistas españolas incluidas en JCR en el decenio 1996-2005

criticar o corregir trabajos anteriores. Otros factores a tener en cuenta son los diferentes grados de citación según las áreas temáticas y sus especialidades, la escasa o nula citación a los trabajos de calidad publicados en revistas de países poco desarrollados, la influencia de las barreras idiomáticas y nacionales y la baja citación de las revistas poco difundidas en bibliotecas y bases de datos $2,3,5,9,12,14$.

\section{"La selección de revistas del SCl no es representativa de la estructura de la producción científica internacional”}

\section{B) Bases de datos SCI y $S S C I$}

Uno de los problemas más serios de estas bases de datos es su sesgo de cobertura a favor de las revistas publicadas en inglés, en detrimento de las procedentes de países no anglófonos, de manera que la selección de revistas del $S C I$ no es representativa de la producción científica internacional. Como resultado, la mayor parte de las citas corresponden a las revistas del área geográfica de la que se han seleccionado las revistas fuente $y$, en consecuencia, los artículos publicados en otros países y escritos en idiomas diferentes al inglés obtienen menos $\operatorname{citas}^{2,13}$. Por lo tanto, el hecho de que una revista esté incluida en la cobertura del $S C I$ favorece que su factor de impacto sea más elevado que el de otras revistas que no lo están. En la figura 1 puede apreciarse que las revistas españolas en $S C I$ se han duplicado en la última década, pasando de 14 en 1996 a 30 en 2005 , mientras que en el SSCI se han mantenido más o menos constantes.
C) Factor de impacto

El FI es una medida global obtenida a partir de las citas que recibe una revista durante un año, pero no todos los artículos han recibido el mismo número de citas, por lo que no es correcto asignar a todos el mismo impacto (algunos estudios demuestran que el 50\% de las citas provienen únicamente del $15 \%$ de los artícu$\left.\operatorname{los}^{9}\right)$. Además, el recuento de citas no distingue entre las que se hacen a artículos de investigación y las que se hacen a otros tipos de artículos de menor importancia, como cartas o editoriales 2, $^{2,9,13,14,27 .}$

Por otra parte, el FI no debe aplicarse para comparar disciplinas diferentes ya que cada una tiene pautas de citación distintas. Favorece a las áreas muy desarrolladas o con gran número de investigadores frente a las pequeñas, lo que determina grandes diferencias entre los factores de impacto de las distintas disciplinas científicas. Por ejemplo, el FI más alto en 2005 en el área Medicine, general \& internal lo obtuvo la revista New England journal of medicine $(\mathrm{FI}=44,016)$, mientras que en el área Information science \& library science la revista más citada fue Mis quarterly (FI = 4,978). De esta diferencia no puede deducirse que los artículos publicados en la primera revista tengan 8,8 veces mayor calidad que los publicados en la segunda.

\footnotetext{
"Los sesgos del factor de impacto del JCR han propiciado el desarrollo de nuevos indicadores alternativos 0 complementarios"
} 


\section{Indicadores alternativos al FI}

Las restricciones del FI han propiciado el desarrollo de nuevos indicadores alternativos o complementarios. Algunos de ellos son ${ }^{6,18-30}$ :

\section{Factor de impacto ajustado (Adjusted IF)•}

Introduce el cálculo de las citas para un periodo de 4 años ${ }^{19}$.

\section{Cited half-life IF}

Sustituye los 2 años de citas en el numerador de la fórmula por la vida media ${ }^{20}$.

\section{Eurofactor $(\boldsymbol{E F})$}

Se presentó en 2002 como la solución al problema de la baja representación de las revistas europeas en el $J C R$, publicando los resultados de 560 revistas europeas clasificadas por especialidades. Se obtiene dividiendo el $\mathrm{n}^{\circ}$ de citas de una revista por el EF-coefficient multiplicando por la raíz cuadrada de la suma del $n^{\circ}$ de citas y del $n^{\circ}$ de artículos ${ }^{21}$.

$$
E F=\frac{\mathrm{N}^{\circ} \text { de citas }}{E F \text {-coefficient } \times \sqrt{\mathrm{n}^{\circ} \text { de citas }+\mathrm{n}^{\circ} \text { de artículos }}}
$$

Su principal limitación es que no revela cuál es su metodología: no indica cuáles han sido sus fuentes de información (revistas analizadas), ni qué citas tiene en cuenta para calcular el Eurofactor, ni si tiene en cuenta las citas de las revistas norteamericanas o las de otros continentes, ni como obtiene el EF coeficient con un valor de 10. Por otra parte, no aparece ni una sola revista española, ni siquiera las que obtienen mayores FI en $J C R^{18,21}$.

Factor de impacto ponderado por especialidad (Disciplinary IF).

Dadas las notables diferencias entre los factores de impacto máximos de cada área, para poder realizar comparaciones entre revistas de áreas científicas diferentes debería utilizarse este factor de impacto ponderado, resultado de dividir el FI de cada revista por el factor de impacto máximo de su área (que corresponde a la primera revista del área con mayor factor de impacto) $)^{22,23}$.

\section{Journal to field impact score}

Incluye artículos de investigación, notas, cartas y revisiones tanto en el numerador como en el denominador de la fórmula del cálculo del FI y un factor corrector por área ${ }^{24}$.

\section{Journal international index (JIN)}

Se basa en el concepto de internacionalidad y en el de revista internacional y para medirla propone las siguientes variables: idioma y país de publicación, inclusión en bases de datos internacionales, FI, afiliación a una asociación o instituto internacional, distribución internacional de los miembros del consejo editorial y de los editores asociados, colaboraciones de autores extranjeros, usuarios multinacionales (lectores, suscriptores y citadores), publicación de trabajos realizados en colaboración internacional y acceso online ${ }^{25}$.

\section{Prestige factor $(P F)$}

En sus orígenes medía el impacto de las revistas científicas incluyendo solamente las citas a artículos originales y 3 años de cobertura. Al parecer, la entidad que lo desarrolló extraía los datos del SCI aplicando un algoritmo, por lo que fueron demandados por el ISI y tuvieron que cerrar por usurpación de la propiedad intelectual ${ }^{26}$.

\section{Factor de Hirsch $(h)$}

El factor $h$ fue propuesto por Jorge E. Hirsch en 2005 y consiste en el $n^{\circ}$ de artículos que tiene un autor con tantas o más citas que su factor $h$. Por ejemplo, un factor h de 20 significa que ese científico tiene 20 artículos que han sido citados 20 o más veces. Las ventajas de este indicador, que se aplica únicamente a los investigadores, son: a) es un promedio extendido a lo largo de los años que sólo puede aumentar o mantenerse estancado; b) se aplica a científicos individuales y permite comparar carreras de científicos de diferentes edades; c) permite extrapolar el rendimiento de un científico a medio plazo ${ }^{27,28}$. Se considera un éxito en la carrera científica de un investigador obtener un h de 20 después de 20 años de trabajo, mientras que un $h$ a partir de 35 sólo se daría en los mejores científicos. El inconveniente que tiene es que, al ser un indicador calculado a partir de las citas, su valor depende del tamaño de la población de científicos que trabajan en un determinado campo, de manera que también está afectado por el sesgo que padecen las áreas poco desarrolladas o con un reducido número de investigadores. Puede aplicarse también a las revistas ${ }^{29}$.

Como se ha visto, la mayor parte de estos indicadores tiene el mismo problema, pues parten de los datos del $S C I$ y $S S C I$ y por lo tanto, de una cobertura no adecuada desde el punto de vista europeo.

\section{"Existe consenso en la necesidad de realizar análisis de citas a partir de las revistas del propio país con el objetivo de obtener indicadores complementarios a los que aporta el JCR"}




\section{Hacia un factor de impacto español}

Hoy en día existe consenso en la necesidad de realizar análisis de citas a partir de las revistas del propio país con el objetivo de obtener indicadores complementarios a los que aporta el $J C R^{7}, 10,12-14,30$. En España en los últimos años han surgido iniciativas con el objetivo de obtener unos indicadores de impacto más adecuados a su realidad, entre ellos, el Factor de impacto potencial de las revistas médicas españolas y el Índice de impacto de las revistas españolas de ciencias sociales (In-Recs), ambas financiadas por diversas convocatorias públicas.

El Factor de impacto potencial de las revistas médicas españolas ha sido desarrollado por el grupo Siniac (Sistemas de información e indicadores de actividad científica) del Instituto de Historia de la Ciencia y Documentación López Piñero, de Valencia. Parte de una población de 103 revistas que cubren todas las especialidades médicas y obtiene cuatro indicadores: el FI e índice de inmediatez nacional y el FI e índice de inmediatez internacional, así como otros datos necesarios para el cálculo de los indicadores, como el número de citas y el de artículos publicados. Para calcular FI e índice de inmediatez nacionales únicamente computan en el numerador de la fórmula las citas que reciben las revistas españolas utilizadas como fuentes, mientras que para determinar el FI y el índice de inmediatez internacionales se añade a las citas anteriores las que las revistas españolas reciben de las extranjeras del JCR. Los indicadores obtenidos, así como los datos utilizados para su cálculo y otros de carácter editorial se presentan en forma de tablas. La figura 2 presenta la página de inicio y la 3 un ejemplo de ficha de una revista.

\section{http://ime.uv.es/imecitas/impacto_ime.asp}

Por otra parte, el grupo de investigación $E C^{3}$ (Evaluación de la ciencia y de la comunicación científica) del Departamento de Biblioteconomía y Documentación de la Universidad de Granada elabora el Índice de impacto de las revistas españolas de ciencias sociales (In-Recs). Parte de una cobertura de 85 revistas de ciencias sociales de las siguientes áreas: antropología, biblioteconomía y documentación, ciencia política y de la administración, comunicación, economía, educación, geografía, sociología, educación y urbanismo. La aplicación permite buscar cuál es el impacto de una revista, su evolución y posición respecto al resto de las revistas de la especialidad, las publicaciones que citan a una revista y las citadas por ella misma, así como los artículos, autores e instituciones más citados de una especialidad. La información se presenta en forma de tablas y gráficas con enlaces hipertextuales entre revistas relacionadas. La figura 4 presenta la página de inicio de la aplicación y la 5 un ejemplo del triángulo de citación de una revista.

http://ec3.ugr.es/in-recs/

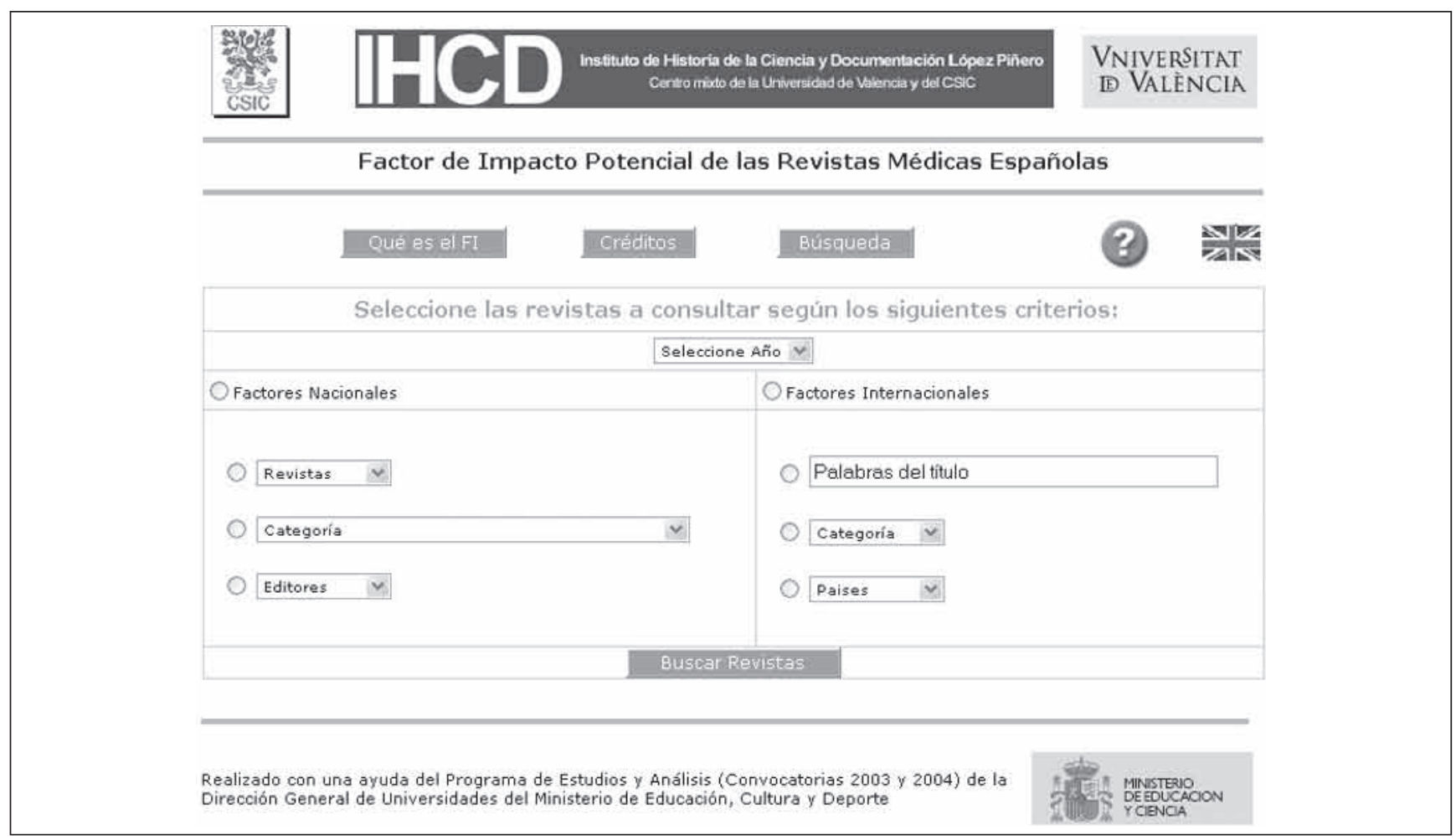

Figura 2. Home page del Factor de impacto potencial de las revistas médicas españolas http://ime.uv.es/imecitas/impacto_ime.asp 


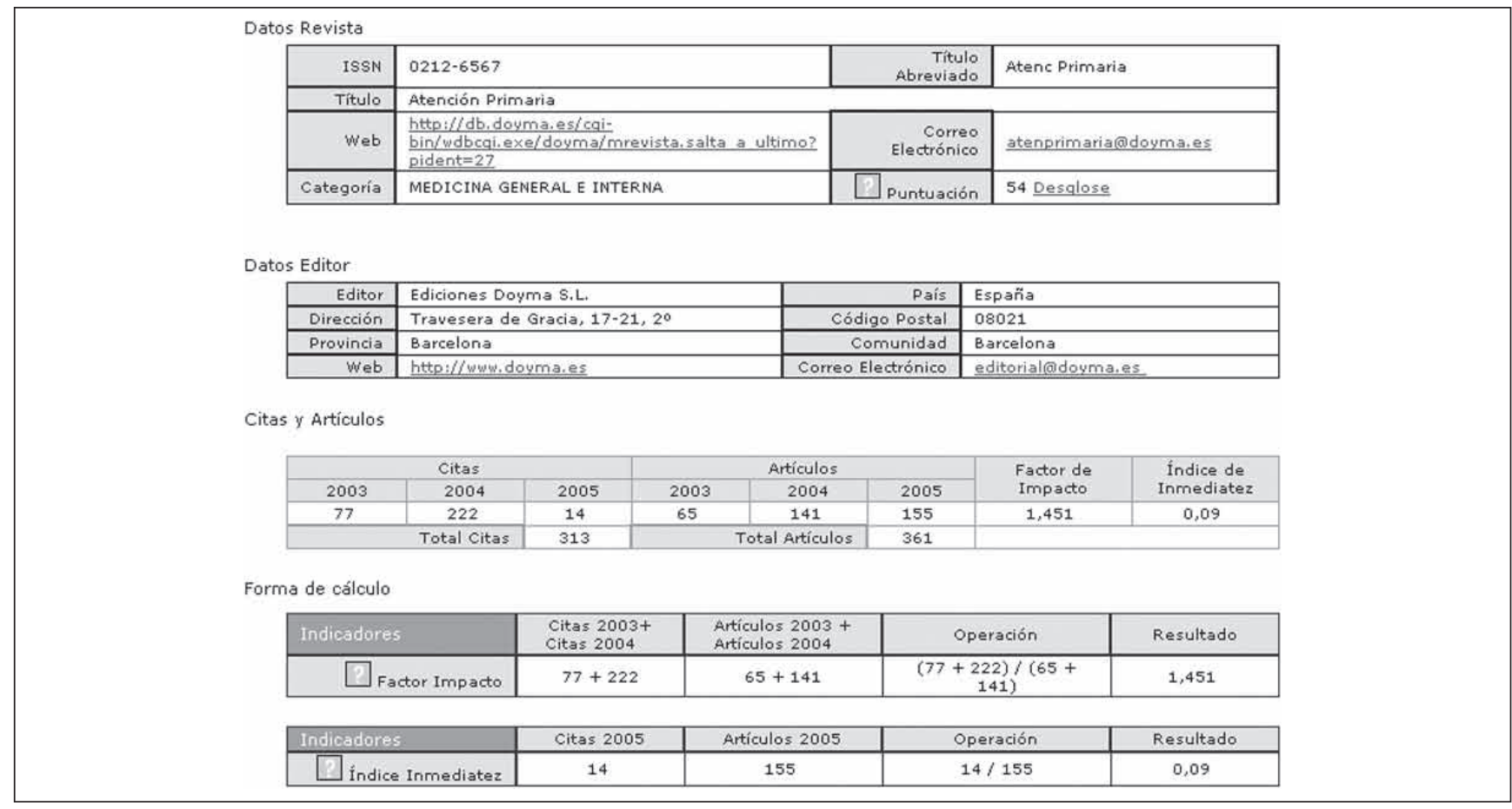

Figura 3. Ficha de una revista del Factor de impacto potencial de las revistas médicas españolas http://ime.uv.es/imecitas/impacto_ime.asp

\section{IN RECS ÍNDICE DE IMPACTO

PEYSTAS ESPANOLASS DE CIENCIAS SOCIALES

\begin{tabular}{|ll}
\hline Antropología & Educación \\
\hline Biblioteconomia y Documentación & Geografía \\
\hline Ciencia politica y de la administración & Sociologia \\
Comunicación & Psicologia \\
Economía & Urbanismo \\
\hline
\end{tabular}

Estadísticas Generales

Qué es

Por qué es necesario

Como se ha confeccionado

A quién puede interesar

Quién lo elabora

Más información

english

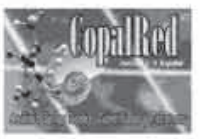

Financiado

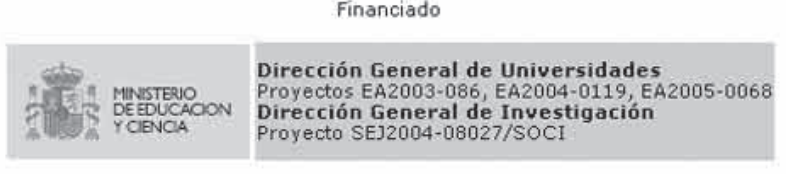

EC $^{3}$

Grupo de investigación

Evaluación de la ciencia y de la comunicación cientifica

Universidad de Granada

Visitas recibidas: 99886

Fecha de publicación: 14/10/2004 Fecha de /a última modificación: 07/03/2006

Optimizada para AS-Explorer con resolución $1024 \times 768$ pixeles

Eniácenos

Estadisticas

Figura 4. Home page de In-Recs (Índice de impacto de las revistas españolas de ciencias sociales) http://ec3.ugr.es/in-recs/ 

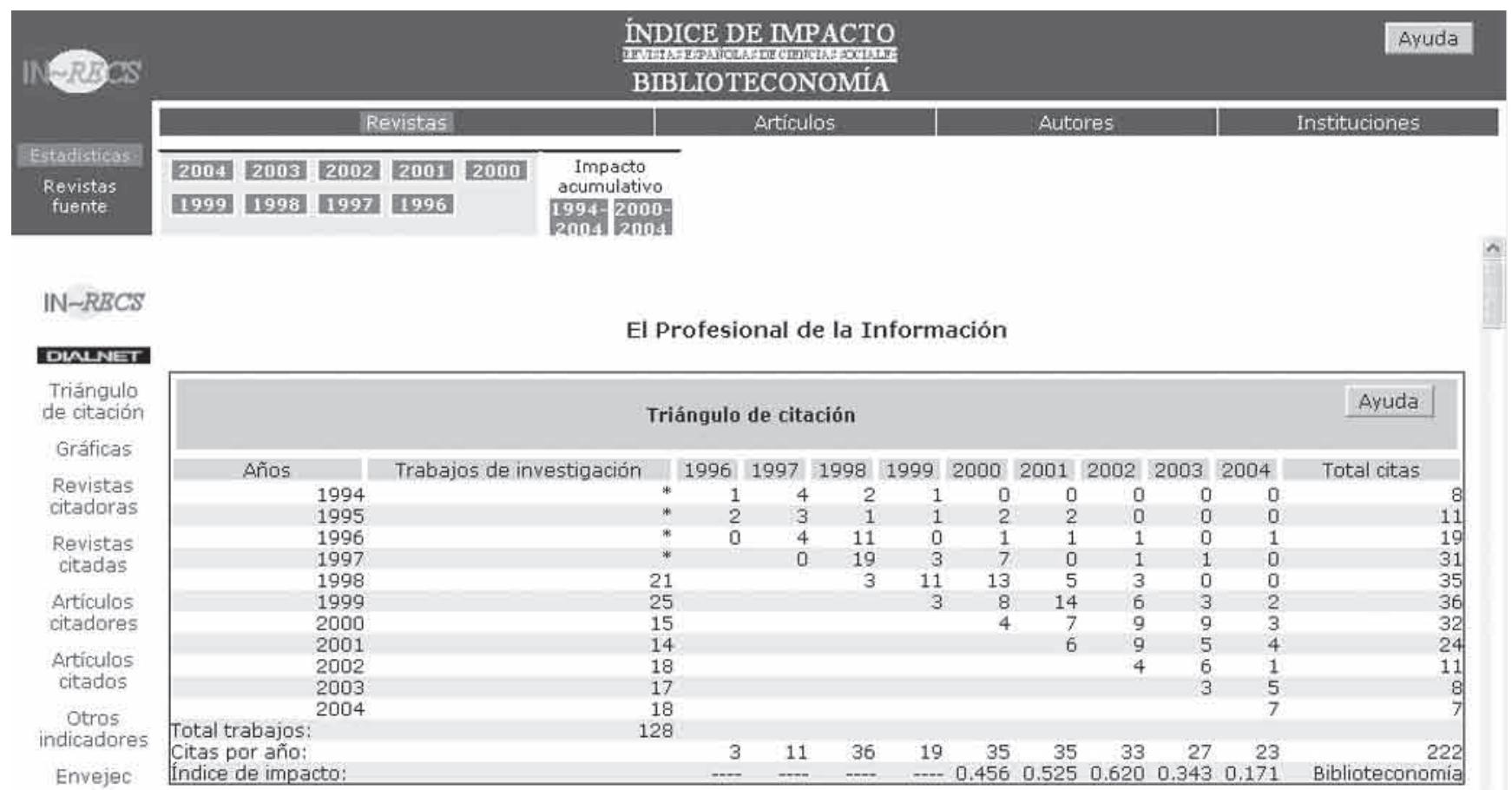

* Sin datos de producción

Figura 5. Triángulo de citación de una revista en In-Recs.

\section{"Entre los indicadores adecuados a la realidad española destacan el Factor de impacto potencial de las revistas médicas españolas y el Índice de impacto de las revistas españolas de ciencias sociales (In-Recs)"}

\section{Conclusiones}

SCI y SSCI tienen importantes limitaciones debidas al excesivo sesgo de cobertura a favor de las revistas norteamericanas y británicas y a la forma de calcular el factor de impacto. Se trata de unas fuentes que excluyen la mayor parte de las revistas que se publican en la Europa Continental y, por lo tanto, los indicadores de impacto que derivan de ellas no pueden proporcionar conclusiones válidas sobre la ciencia que se desarrolla en esta zona ${ }^{13}$. Su uso en la evaluación de la investigación ha sido criticado en numerosas ocasiones, tanto entre los profesionales, que ven frustradas sus expectativas de promoción profesional, como en el periodismo científico español, que acude impotente a la exportación gratuita de la ciencia española a las revistas extranjeras, sobre todo a las angloamericanas del área de cobertura de SCI y SSCI.

Los criterios que equiparan repercusión con calidad son, en algunos casos, falsos y engañosos. El factor de impacto no es una medida directa de la calidad de una revista, sino de la frecuencia con la que son citados sus artículos en un año $0^{6-9,11,22}$. La valoración de la calidad exclusivamente a través del impacto es incorrecta, ya que se puede encontrar ciencia de primera calidad en revistas de escasa difusión o en publicaciones no incluidas en $S C P^{31}$.

El uso indiscriminado del factor de impacto como medida de evaluación de los investigadores puede provocar cambios en las costumbres de éstos, pues tenderán a evitar la investigación que no sea rentable bibliométricamente (investigación aplicada y sobre temas locales) y puede desembocar en una picaresca en la citación con la finalidad de aumentar el impacto (abuso de autocitas, citación de resúmenes sin haber leído los artículos o establecer sistemas de cita mutua con otros colegas $\left.^{13}\right)$.

Las iniciativas llevadas a cabo para elaborar índices de citas y determinar indicadores de repercusión a partir de las revistas españolas no tienen los sesgos de las bases de datos extranjeras y permiten identificar las revistas españolas de mayor calidad desde el punto de vista de la investigación desarrollada y publicada en España.

\section{"Los criterios que equiparan repercusión con calidad son, en algunos casos, falsos y engañosos"}


Los bajos valores de los indicadores obtenidos por las revistas españolas ponen en evidencia la necesidad de mejorar su impacto adoptando medidas que aumenten su calidad, el nivel científico de los artículos, promover la internacionalización de las revistas, incluir resúmenes más amplios en inglés, realizar ediciones bilingües en inglés y castellano, y fomentar la fusión de determinadas revistas ${ }^{30}$.

\section{Bibliografía}

1. De la Cueva Martín, A.; Aleixandre-Benavent, R.; Rodríguez Gairín, J. M. Manual de fonts d'informació en ciències de la salut. Valencia: Universitat de València, 2001, ISBN: 84-370-5145-2.

2. Aleixandre Benavent, R.; Porcel Torrens, A. "El factor de impacto de las revistas científicas". En: Trastornos adictivos, 2000, v. 1, n. 4, pp. 264-271.

3. López Piñero, J. M.; Terrada, M. L. "Los indicadores bibliométricos y la evaluación de la actividad médico-científica. (II). La comunicación científica en las distintas áreas de las ciencias médicas”. En: Medicina clínica 1992, v. 98, pp. 101-106.

4. Baños, J. F.; Casanovas, L.; Guardiola, E.; Bosch, F. "Análisis de las revistas biomédicas españolas mediante el factor de impacto". En: Medicina clínica, 1992, v. 99, pp. 96-99.

5. De Granda Orive, J. J. "Algunas reflexiones y consideraciones sobre el factor de impacto". En: Archivos de bronconeumología, 2003, v. 39. pp. 409-417.

6. González de Dios, J.; Mateos Hernández, M. A.; González Casado, I. "Factor de impacto internacional, nacional y por especialidades: en busca del mejor indicador bibliométrico". En: Revista española de pediatría, 1998 , v. 54 , pp. $430-436$

7. Garfield, E. Ciation indexing. Its theory and application in science, technology and humanities. Nueva York, John Wiley and Sons, 1979.

8. Seglen, P. O. "How representative is the journal impact factor?". En: Research evaluation, 1993, v.2, pp. 143-149.

9. Seglen, P. O. "Why the impact factor of journals should not be used for evaluating research". En: British medical journal, 1997, v. 314, pp. 498502. Consultado el 13-11-2006:

http://bmj.bmijournals.com/cgi/content/full/314/7079/497

10. Stegmann, J. "How to evaluate journal impact factors". En: Nature, 1997, pp. 390(6660):550.

11. Amin, M.; Mabe, M. "Impact factor: use and abuse". En: Perspectives in publishing, 2000, v. 1, pp. 1-6.

12. Bordons, M.; Fernández, M. T.; Gómez, I. "Advantages and limitations in the use of impact factor measures for the assessment of research performance". En: Scientometrics, 2002, v. 53, pp. 195-206.

13. López Piñero, J. M.; Terrada, M. L. "Los indicadores bibliométricos y la evaluación de la actividad médico-científica. (I). Usos y abusos de la bibliometría" En: Medicina clínica, 1992, v. 98, pp. 64-68.

14. González de Dios, J.; Moya, M.; Mateos Hernández, M. A. "Indicadores bibliométricos: características y limitaciones en el análisis de la actividad científica". En: Anales españoles de pediatría, 1997, v. 47, pp. 235-244.

15. Kaplan, N. "The norms of citation behaviour. Prolegomena to the footnote". En: American documentation, 1965, v. 16, pp. 179-184.
16. Merton, R. K. "Behaviour patterns of scientists". En: The American scholar, 1969, v. 38, pp. 197-225.

17. Alfonso, F.; Bermejo, J.; Segovia, J. "Impactología, impactitis, impactoterapia”. En: Revista española de cardiología, 2005; v. 58, pp. 1239-1245.

18. Peng Dong, M. L.; Mondry, A. "The impact factor revisited". En: Biomedical digital libraries, 2005, v. 2, p. 7. Consultado el 12-11-2006:

http://www.bio-diglib.com/conetnt/2/1/7

19. Asai, I. "Adjusted age distribution and its application to impact factor and immediacy index". En: Journal of the American Society for Information Science, 1981, v. 32, pp. 172-174.

20. Sombatsompop, N.; Markpin, T.; Premkamolnetr, N. "A modified method for calculating the impact factors of journals in ISI Journal citation reports: Polymer science category in 1997-2001”. En: Scientometrics, 2004, v. 60 , pp. $217-35$.

21. Hofbauer, R.; Frass, M.; Gmeiner, B.; Kaye, A. D. (eds.). Euro-Fac$\operatorname{tor}(E F)^{T M}$. The European journal quality factor. The new European "scientific currency". Vienna: Vicer Publishing, 2002. Consultado el 12-11-2006. http://www.univ-lille1.fr/lea/Menu_du_Site/Publications/Acrobat/VICEREUROFACTOR.pdf

22. Ramírez, A. M.; García, E. O.; Río, J. A. D. "Renormalized impact factor". En: Scientometrics, 2000, v. 47, pp. 3-9.

23. Hirst, Graeme. "Discipline impact factors: a method for determining core journal lists". En: Journal of the American Society for Information Science, 1978, v. 29, pp. 171-172

24. Leeuwen, T. N. van; Moed, H. F. "Development and application of journal impact measures in Dutch science system". En: Scientometrics, 2002, v. 53 , pp. 249-266.

25. Buela-Casal, G.; Perakakis, P.; Taylor, M.; Checa, P. "Measuring internationality: reflections and perspectives on academic journals". En: Scientometrics, 2006, v. 67, pp. 45-65.

26. Buela-Casal, G. "La evaluación de la investigación científica: el criterio de la opinión de la mayoría, el factor de impacto, el factor de prestigio y 'Los diez mandamientos' para incrementar las citas". En: Anales y modificación de la conducta, 2002, v. 28, pp. 455-476.

27. Hirsch, Jorge E. "An index to quantify an individual's scientific research output". En: Proceedings of the National Academy of Sciences of the United States of America, 2005, v. 102. pp. 16.569-16.572.

28. Grupo Scimago. "El índice h de Hirsch: aportaciones a un debate". En: El profesional de la información, 2006, v. 15, n. pp 304-306.

29. Braun, T.; Glänzel, W.; Schubert, A. "A Hirsch-type index for journals". En: The scientist, 2005, v. 19, n. 22, pp. 8.

30. Aleixandre-Benavent, R.; Valderrama Zurián, J. C.; Miguel-Dasit, A.; Alonso Arroyo, A.; Castellano, M. "Hypothetical influence of non-indexed Spanish medical journals on the impact factor of the Journal Citation Reports-indexed journals". En: Scientometrics (en prensa).

31. Aleixandre, R.; Valderrama, J. C.; Desantes, J. M.; Torregrosa, A. J. Identification of information sources and citation patterns in the field of reciprocating internal combustion engines. En: Scientometrics, 2004, v. 59, n. 3, pp. 321-336.

Rafael Aleixandre-Benavent, Juan Carlos Valderrama-Zurián y Gregorio González-Alcaide, Instituto de Historia de la Ciencia y Documentación López Piñero. Avda. Blasco Ibáñez, 15. 46010 Valencia.

aleixand@uv.es

juan.valderrama@uv.es

gregorio.gonzalez@uv.es 


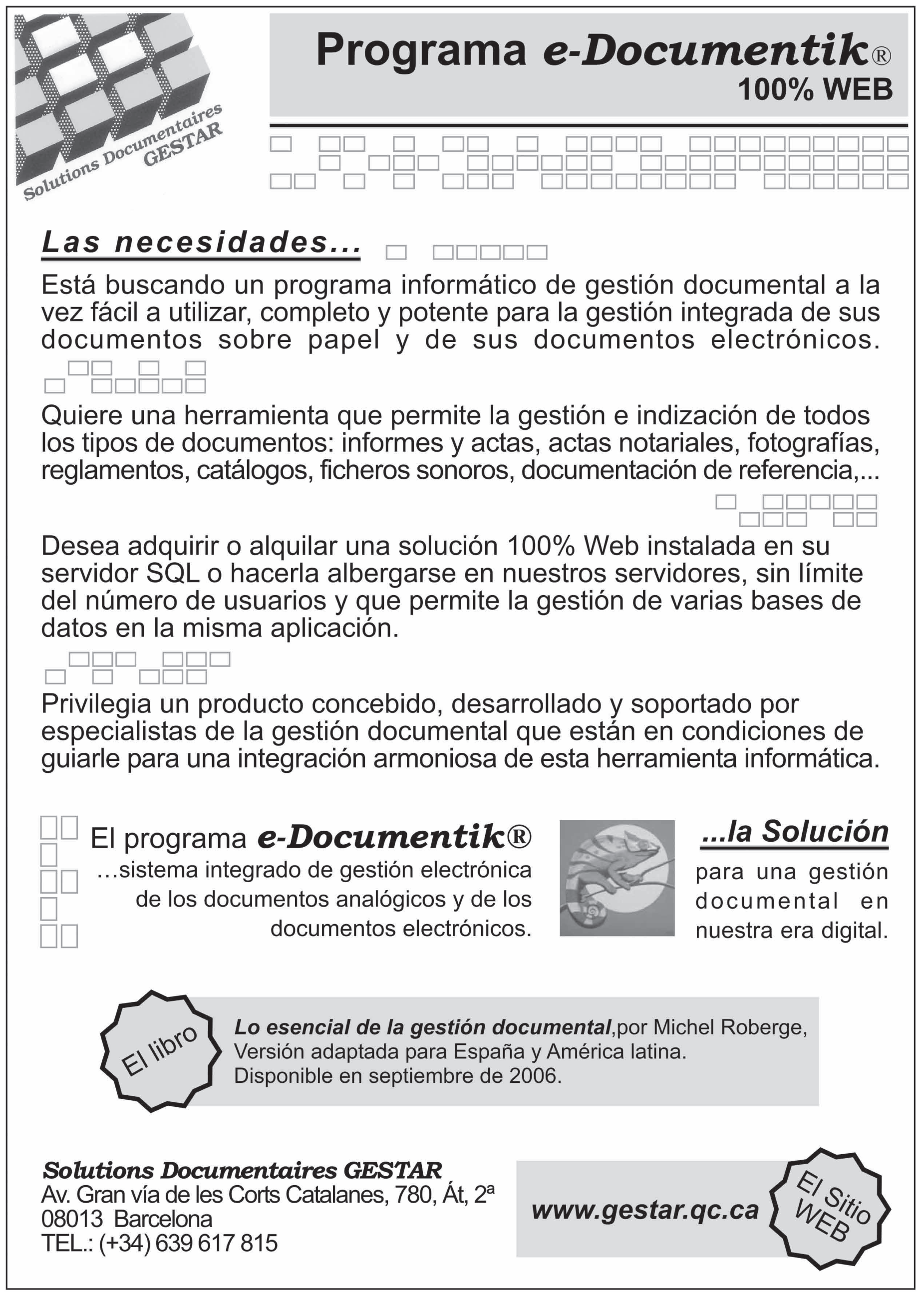

